\title{
Sostenibilidad en la cadena de suministro: evidencias del sector minorista español
}

\section{Sustainability in the supply chain: evidence from the spanish retail sector}

\author{
Jesús Morcillo-Bellido \\ Universidad Carlos III de Madrid \\ jesus.morcillo@uc3m.es
}


La "sostenibilidad» en la cadena de suministro constituye uno de los retos importantes del siglo XXI. Este trabajo tiene como objetivo profundizar la comprensión de cómo la sostenibilidad integral puede ser fomentada, desarrollada y soportada a través de alianzas y prácticas de lean operations en una cadena de suministros del sector de Servicios. A través del análisis realizado se busca determinar en qué medida se ha conseguido alcanzar un alto nivel de sostenibilidad integral en la cadena de suministro estudiada, soportado por su propio modelo de alianzas y la aplicación sistemática y estricta de prácticas de lean operations. La cadena de suministro objeto de estudio presenta, además, la particularidad de buscar que las prácticas de eficiencia desarrolladas en la empresa focal (Mercadona) sean ampliamente replicadas en sus más de cien proveedores clave. Adicionalmente, la empresa focal está impulsando la extensión de estas prácticas a los proveedores de materias primas para alcanzar la sostenibilidad en la totalidad de su cadena. La principal contribución de este estudio es que intenta mostrar cómo la sostenibilidad integral en la cadena de suministro puede encontrar soporte para su desarrollo en elementos estratégicos que, bajo ciertas condiciones, pueden formar un sólido modelo integral, potencialmente aplicable a determinadas cadenas de suministro en sectores con características similares.

Palabras clave: sostenibilidad, cadena de suministro, alianzas, interproveedores, prácticas lean

"Sustainability" in the supply chain is one of the important challenges of the $21 \mathrm{st}$ century. This work aims to deepen the understanding of how integral sustainability can be fostered, developed and supported through alliances and lean practices operations in a supply chain of the Services sector. Through the analysis carried out, it is sought to determine to what extent it has been possible to achieve a high level of comprehensive sustainability in the analyzed supply chain, supported by its own alliance model and the systematic and strict application of lean operations practices. The supply chain studied also presents the peculiarity of seeking that the efficiency practices developed in the focal company (Mercadona) are widely replicated in its more than one hundred key suppliers. The focal company is promoting the extension of these practices to its raw materials suppliers to achieve sustainability in the whole chain. The main contribution of this study is that it tries to show how integral sustainability in the supply chain can be supported for its development in strategic elements that, under certain conditions, could create a solid integral model, potentially applicable to certain supply chains in sectors with similar characteristics.

Keywords: sustainability, supply chain, alliances, interproviders, lean practices 


\section{Introducción}

La expresión "sostenibilidad», entendida como integración de los aspectos relacionados con los resultados económicos, los aspectos sociales y el impacto medioambiental en la gestión de las organizaciones, comenzó a surgir en la literatura económica, y en particular en temas relacionados con el estudio de las operaciones y la cadena de suministro, en los últimos años del siglo pasado (Carter \& Rogers, 2008). Su aparición fue, en gran medida, un reflejo de la visión de sostenibilidad desarrollada por la Brundtland Commission (1985), que define "desarrollo sostenible» como la capacidad para "satisfacer las necesidades de las generaciones presentes sin comprometer las posibilidades de las del futuro para atender sus propias necesidades".

Esos tres aspectos fueron integrados en un concepto desarrollado por Elkington $(1994,1998,2004)$ que se ha denominado the triple bottom line, el cual corresponde con la idea de tener siempre en mente los tres resultados (económico, social y medioambiental) en la gestión integral de cualquier empresa del siglo XXI. En esa línea, solo las organizaciones que consiguen un correcto equilibrio en la dirección de sus actividades para que estas conduzcan a buenos resultados económicos, sociales y medioambientales pueden considerarse como "organizaciones sostenibles».

Autores como Forrester (1958), La Londe y Masters (1994), Cooper, Ellram, Gardner y Hanks (1997), Lambert y Cooper (2000), y Chopra y Meindl (2004) desarrollaron y extendieron el término "cadena de suministro" como la coordinación entre un conjunto de organizaciones para conseguir que el flujo de materiales de un determinado bien sea eficiente en términos de servicio y coste. Eso implica que las organizaciones se involucren en el aprovisionamiento, la producción y la distribución de los materiales necesarios para la realización de dicho bien, así como en el transporte del mismo, una vez terminado, hasta el consumidor final, siendo todas estas organizaciones elementos de la misma cadena de suministro (Mentzer et al., 2001; Coyle, Langley, Novack \& Gibson, 2016; Mangan, 2016; Sindi, 2017). Otros autores, como Christopher (2016), definen la cadena de suministro como un conjunto de procesos que generan valor en el flujo de bienes y servicios hasta que estos Ilegan al usuario final (De la Calle, Barinaga \& Gietz, 2016).

Con esta visión de las cadenas de suministro resulta imprescindible no solo integrar prácticas de dirección que promuevan la consecución de los objetivos «tradicionales» de la propia cadena, tales como el coste, la calidad en el servicio y la fiabilidad, sino que deben ir más allá, enfocándose no solo en la consecución de los aspectos económicos que se establezcan, sino también en los aspectos sociales y medioambientales (Carter \& Rogers, 2008; Dubey et al., 2017).

Kolk (2008) publicó un estudio basado en más de 3000 entrevistas online llevadas a cabo con ejecutivos de empresas de tamaño medio/grande distribuidas por todo el mundo, donde se les preguntaba sobre las prácticas de sostenibilidad que esas empresas desarrollaban y, sobre todo, cuáles eran los temas relacionados con la sostenibilidad que tenían más prioridad para sus compañías. De las respuestas reunidas en este estudio se deduce que los tres temas principales en los que las empresas están trabajando para conseguir prácticas empresariales más sostenibles son: 
- Reducción del consumo de energía en la cadena de suministros (66\%).

- Reducción de los desperdicios en la cadena de suministro (64\%).

- Adopción de políticas y acciones relacionadas con la responsabilidad social corporativa (57\%).

El segundo aspecto está muy relacionado con las prácticas que se han incluido en los últimos años bajo el término lean operations, ya que el concepto de lean desarrollado por Womack y Jones (2010) ha generado en las últimas décadas un gran interés al poner énfasis en la eliminación de desperdicios en las organizaciones. Womack y Jones entienden como desperdicio todo uso de recursos o actividades que no aportan valor al cliente, y su eliminación supone ir un paso más allá del llamado lean manufacturing (Womack, Jones \& Roos, 1990), que puede considerarse como la extensión a todo tipo de organizaciones, tanto industriales como de servicios, de los conceptos y prácticas desarrolladas bajo la filosofía de operaciones de Toyota (Ohno, 1988; Rother, 2017). En general, ha sido en la industria donde más se han aplicado estas prácticas y el área de los servicios sigue siendo todavía un campo bastante inexplorado para ellas, quizás porque las organizaciones de este sector no han entendido todavía su utilidad y aplicabilidad real. Solo algunas empresas relacionadas con la salud, las telecomunicaciones y los seguros han comenzado a desarrollar proyectos basados en prácticas lean.

Algunos autores (Govindan, Azevedo, Carvalho \& Cruz-Machado, 2014) consideran que los aspectos relacionados con lean y resiliencia, entendida esta como la capacidad de recuperación ante un acontecimiento negativo inesperado (Sheffi, 2005), son aspectos críticos que, si son manejados de una forma apropiada, permiten a las compañías ser más sostenibles de forma integral, especialmente cuando las mismas se encuentran en una situación en la que el mercado es altamente volátil, como en la realidad empresarial actual.

En este trabajo se considera que algunas organizaciones han llevado a cabo una combinación de los aspectos anteriores con otros que les son particulares y forman parte de su estrategia empresarial, buscando ir un poco más allá en la construcción de cadenas de suministro sostenibles. Así, la cadena de suministro formada por el líder en distribución minorista en España (Mercadona) y sus más de 100 «interproveedores» podría ser un ejemplo de cadena de suministro sostenible que va más allá de lo habitualmente descrito como tal. En un estudio previo (Morcillo \& Duran, 2016) se analizaron las relaciones colaborativas (alianzas) entre Mercadona y su red de principales proveedores (interproveedores), concluyendo que factores como la existencia de claros "objetivos estratégicos compartidos" y de "objetivos a largo plazo" en las relaciones de colaboración supone un valor añadido que explica el éxito superior de las alianzas entre Mercadona y sus interproveedores respecto a otras organizaciones, que también llevan a cabo un número importante de alianzas, pero no han conseguido desarrollar mecanismos tan fuertes de relación a largo plazo. Estos dos factores cualitativos aportarían, en el caso de Mercadona, un "valor adicional» especial en las relaciones de colaboración con sus socios, con la particularidad de que además se establecen «de por vida». Todos estos mecanismos podrían considerarse un ejemplo de desarrollo de capacidades en alianzas (Morcillo, Díaz \& Solís, 2015). 


\section{Objetivos del estudio}

Según Ketokivi y Choi (2014), una de las principales características de la investigación es la transparencia, de tal forma que se pueda comprender la lógica del análisis que conduce a unas determinadas conclusiones y las premisas que las sustentan. El estudio de casos empresariales es, justamente, una forma adecuada para llevar a cabo investigaciones científicas que cubran todos los requisitos que se requieren para conseguir la citada transparencia. En este trabajo se pretende profundizar en el conocimiento de cómo unas determinadas prácticas estratégicas influyen sobre algo que va más allá de la estrategia habitual en las organizaciones, como es la sostenibilidad.

Con este trabajo se propone dar un paso adicional hacia el estudio del modelo de sostenibilidad de la cadena de suministro de este minorista (Mercadona), considerando que la sostenibilidad desarrollada en su cadena de suministro podría explicarse por la construcción de un "conjunto armónico» de factores que distinguen su cadena de suministro de otras, tales como:

- Factores tradicionales que explican la formación de alianzas, que también se producen en otras cadenas de suministro.

- Factores adicionales que fortalecen sus relaciones de colaboración en las empresas que poseen los mencionados objetivos compartidos a largo plazo.

- La utilización de prácticas de lean operations, tanto en Mercadona como en su red de interproveedores, que apoyan la sostenibilidad en las cadenas de suministro, tal como fueron estudiadas en los trabajos desarrollados por Wee y Wu (2009).

- La reproducción del modelo desarrollado por Mercadona en las prácticas internas de sus principales interproveedores, los cuales han puesto en marcha mecanismos que actúan como factores multiplicadores, provocando la reproducción de la forma de trabajo desarrollada por la propia organización del distribuidor (Morcillo \& Duran, 2017). Esto, a su vez, estaría retroalimentando la cadena integral y reforzándola a través de un "proceso de mejora continua».

\section{Metodología}

Para llevar a cabo este estudio prospectivo, que explora un tema que aún no está suficientemente desarrollado, se han utilizado principalmente métodos de investigación cualitativa (entrevistas semiestructuradas y análisis de datos publicados) que destacados autores (Eisenhardt, 1989; Yin, 2013; Gummesson, 1991) recomiendan como prácticas adecuadas para la exploración de los aspectos innovadores de gestión estratégica de la organización.

La información ha sido obtenida a través de: a) entrevistas en profundidad con ejecutivos de alto nivel pertenecientes a tres importantes interproveedores de Mercadona, y b) el análisis de los datos publicados (incluido el de la información disponible en la web y en foros, conferencias, trabajos publicados, etcétera). 
En cuanto a las entrevistas en profundidad, se ha entrevistado a cinco ejecutivos de los tres principales interproveedores: interproveedor $\mathrm{A}$ (director general y director de operaciones), interproveedor B (director general) e interproveedor C (director de marketing y director de operaciones). Para seleccionar estas tres compañías se buscó que sean parte de los 20 principales interproveedores de Mercadona y que hubiese facilidad de acceso a sus ejecutivos. Cabe destacar que, para alcanzar el objetivo de este estudio, fue importante que estuvieran dispuestos a discutir sobre aspectos sensibles de sus organizaciones, como su estrategia y sus prácticas en la cadena de suministro.

Todas las entrevistas se realizaron entre los años 2016 y 2017, usando encuestas semiestructuradas con un mínimo de dos reuniones por persona, así como las preguntas tratadas, se centraron principalmente en recoger sus experiencias y opiniones sobre los aspectos tratados en los objetivos del estudio. En todos los casos, los entrevistados recibieron un breve cuestionario previo para que tuvieran la posibilidad de revisar las preguntas con tiempo suficiente.

\section{Análisis de la cadena de suministro}

Mercadona es actualmente el líder en el mercado español de distribución minorista (solo distribuye una limitada gama de productos de alimentación, limpieza del hogar y comida para mascotas). Sus ventas alcanzaron un valor de € 21623 millones en el año 2016, con una participación de mercado del 23,4\% y más de 79000 empleados en 1614 tiendas. Solo opera en España y en 2019 abrirá sus primeras tiendas en Portugal.

El negocio de Mercadona ha crecido rápidamente en los últimos años al haber encontrado una forma eficiente de proporcionar a los consumidores los productos que demandan a un precio que estos consideran atractivo. En el año 1993, después de rechazar potenciales ofertas de compra de la empresa, el dueño decidió implantar lo que denominó el «modelo de Calidad Total» como la base de todas las operaciones en la organización (Tan, Wong \& Choong, 2015).

La compañía pasó de trabajar dentro de un modelo de precios variables, adaptable continuamente a la demanda y a los precios de la competencia, común en la industria y basado en las promociones, a un modelo donde los clientes podían encontrar "siempre precios bajos (SPB)» dentro de una estrategia caracterizada por la calidad y la mejora continua (Ton \& Harrow, 2010). Mercadona dejó de hacer promociones y mantuvo su compromiso de precios estables con sus clientes, basándose en acuerdos a largo plazo con sus proveedores. Este cambio fue una auténtica revolución en el modelo de negocio, ya que el anterior — que podemos denominar como «tradicional»— se basaba en mantener la rentabilidad de la empresa minorista, trabajando los tres ejes que la industria minorista había considerado hasta ese momento como la base de la rentabilidad del conjunto de la industria, si se manejaban con suficiente flexibilidad. El modelo tradicional indicaba que los precios debían de ser lo más altos posible (ofreciendo promociones en ciertos productos considerados como "ganchos»), que a los proveedores se les debía de presionar, renegociando los acuerdos cada día, y que a los empleados se les debía mantener dentro de un esquema continuo de rotación y cambio de horarios. 
A la vez que introducía un modelo de precios bajos estables, la compañía puso en marcha todas aquellas medidas que podían reducir el "desperdicio; es decir, todo aquello que no aportaba valor a sus clientes. Una de las medidas para ello fue el desarrollo de «marcas del distribuidor» (MDD), donde un proveedor fabricaba productos con la marca propia de Mercadona y este las comercializaba con un descuento de 20-30\% respecto a la marca del fabricante líder en la categoría (Miguel \& Santiago, 2010).

El fenómeno de las MDD o marcas del distribuidor no es una estrategia exclusiva de Mercadona, ya que la inmensa mayoría de sus competidores también ha optado por aplicarla en España. Desde Lidl, cuya marca propia representó en 2014 casi el 79,6\% de sus ventas en España, hasta Alcampo, que tiene un 19,3\% de su cifra de negocio atribuible a marcas propias, las MDD son usadas como estrategia para evitar el conocido efecto «doble margen», ya que tanto el fabricante como el detallista tienden a cargar un margen adicional al valor añadido que proporcionan a sus clientes (Durhan, 2000; Gaudin, 2016; Hong, 2017). Mercadona utilizó esta práctica para desarrollar productos con su marca que eran fabricados por ciertos proveedores seleccionados, que además cumplían la condición de no fabricarla para otros competidores y, generalmente, también renunciaban a comercializarlos con su propia marca.

Los clientes (los jefes, en la terminología propia de la empresa) son el centro de un modelo soportado por una importante implicación de los empleados y de los principales proveedores (126 interproveedores en 2017), quienes garantizan que estos reciban los productos que Mercadora, a partir de un minucioso análisis, ha determinado como los necesarios en la cesta de la compra de sus clientes, con total garantía de calidad y seguridad alimentaria.

El aspecto de la seguridad alimentaria es especialmente cuidado por la empresa y considerado como un factor básico para garantizar la calidad en toda la cadena de suministro. Esta calidad en la forma de trabajar (procesos) debe garantizar la seguridad alimentaria e incluye tanto el cumplimento de las normas legales que aseguran una perfecta higiene en los procesos productivos como la existencia de normas internas propias. Y todo ello dentro de un enfoque de "cero desperdicios".

Cada uno de los elementos incluidos en este modelo tiene sentido en la ejecución del servicio comprometido con el cliente. En el periodo 2016-2018, Mercadona pretende dar un paso más para mejorar la coordinación de la cadena de suministro al integrar a los proveedores de productos frescos, tales como lonjas y agricultores, dentro de un proyecto denominado Caspopdona. Esto significa que se busca integrar todos los eslabones de la cadena de suministro, yendo en la búsqueda de la mejora y el control de la misma desde el proveedor de las materias (el agricultor en el caso del trigo o el barco pesquero en el del pescado) hasta el consumidor final. Esta integración es un caso único entre las cadenas de suministro de las diferentes cadenas detallistas españolas y no existen precedentes de este tipo de prácticas en el sector.

La integración busca garantizar que, aguas arriba en la cadena de suministro de Mercadona, existen proveedores mejorando sus productos y sus procesos para que los clientes finales reciban productos con una alta calidad y seguridad alimentaria, consiguiendo además un flujo de suministro regular a precios más estables que los del mercado. 
Un caso que la propia compañía cita como ejemplo de interproveedor en su cadena de suministro sostenible es el del buque pesquero Elvimar, con base en Barbate (Cádiz), que surte junto a otros 47 buques una parte importante de la cadena de suministro de pescado fresco de Mercadona. La integración constituye así un paso innovador que incluso cabe catalogar como revolucionario en una industria en la que, hasta ahora, lo que ha primado es la falta de coordinación y objetivos comunes entre distribuidores y proveedores. Este nuevo modelo de negocio busca la innovación constante a través de la cadena de suministro y tiene como objetivo final conseguir una "cadena de suministro sostenible» (Ehrenfeld, 2004) en los términos que considera Mercadona. Al mismo tiempo, la empresa está convencida de la oportunidad que este modelo significa para sentar las bases de un crecimiento del sector primario y permitir que cuantos aportan valor en la cadena de suministro también ganen, reforzando con ello el equilibrio social entre todos los eslabones.

\subsection{Modelo de mejora continua en red}

El compromiso de calidad de Mercadona con sus clientes está estrechamente vinculado al compromiso a largo plazo que mantiene con sus proveedores y al riguroso cumplimiento de las normas de seguridad alimentaria que aplica en su cadena de suministro, el cual exige a todos aquellos que quieren participar de ella, tal como se ha comentado anteriormente. Considera categorías de proveedores, según la mayor o menor vinculación con los mismos, el compromiso a largo plazo y el impacto esperado en su cadena de suministro. En la tabla 1 están identificados los tipos de proveedores que distingue la compañía.

\section{Tabla 1.Tipos de proveedores}

\begin{tabular}{l|l} 
tipo de proveedor & características \\
\hline $\begin{array}{l}\text { Interproveedor } \\
\text { (126 empresas) }\end{array}$ & $-\quad$ Enfocado a una categoría de productos \\
& $-\quad$ En general, fabrica la marca del distribuidor \\
& $-\quad$ Suministra a Mercadona la totalidad o una parte superior a 2/3 de su \\
& $-\quad$ Trabaja bajo las normas y el control de Mercadona \\
& $-\quad$ Mantiene relaciones basadas en criterios open book \\
& $-\quad$ Tiene una relación de alianza de por vida \\
\hline $\begin{array}{l}\text { Proveedor especialista } \\
\text { (30-40 empresas) }\end{array}$ & $-\quad$ Suministra un producto específico en el que está especializado \\
\hline $\begin{array}{l}\text { Proveedores } \\
\text { (2000 empresas) }\end{array}$ & $-\quad$ Es el resto de suministradores contratos a largo plazo ni relación de alianza \\
\hline
\end{tabular}

Fuente: elaboración propia.

Mercadona emplea un sistema de categorización de proveedores en el que destaca un grupo de élite, los «interproveedores», que están perfectamente integrados al modelo de Mercadona y actualmente forman un conjunto de 126 empresas, cuyos procesos productivos están también totalmente integrados a la cadena de suministro de 
la empresa focal. Como ejemplo de este modelo cabe citar las alianzas con Covap (leche), Grupo Siro (galletas) y grupo Tarradellas (pizzas y embutidos).

$\mathrm{Al}$ analizar las competencias que estos interproveedores han desarrollado en esta relación se pueden mencionar algunas muy destacadas (figura 1): a) muy alto enfoque hacia la excelencia en la calidad como consecuencia de los estrictos requisitos y normas de su cliente; b) control estricto de sus procesos, ya que en la estrategia que Mercadona aplica en su relación con sus interproveedores no hay lugar para el más mínimo desperdicio que pueda significar un mayor coste y la subsiguiente repercusión en el precio final, razón por la cual los proveedores son obligados a analizar de una forma sistemática las potenciales áreas perfectibles para aplicar acciones que continuamente mejoren los productos que ofrecen; c) innovación permanente, tanto en los procesos como en los productos elaborados; d) establecimiento de alianzas con sus proveedores bajo el mismo modelo establecido entre Mercadona y sus interproveedores; y e) colaboración en el plan para integrar en la cadena de suministro a los proveedores de las materias primas que se incorporan en los productos finales (cereales, piensos, etcétera) o que son vendidas como productos frescos al consumidor (proyecto Caspopdona). Esto obliga a los interproveedores a desarrollar un trabajo aguas arriba, en sus propias cadenas de suministro, con el fin de establecer unos estándares de calidad en los procesos de sus proveedores, práctica que supone un esfuerzo a la vez de colaboración y de control.

Resultado de la iniciativa (Caspopdona) es el proyecto pionero Girasoles, que ha contado con la participación de 2800 ganaderos. Prueba de su éxito son las 690 000 cabezas de ganado porcino anuales con las que más de 140 ganaderos nacionales diferentes abastecen a Casa Tarradellas; o la producción, superior a los 370000 litros de leche, obtenida por 1400 ganaderos para los interproveedores lácteos Lactiber, Covap e Iparlat; o los acuerdos de producción y suministro de arroz suscritos por Arrocerías Pons con 26 agricultores de cinco comunidades autónomas.

Respecto a la calidad, ambos (Mercadona y sus interproveedores) están comprometidos en una doble garantía al consumidor, rotulando los interproveedores su propia marca en los envases como prueba del compromiso asumido. Mercadona quiere que el comprador sepa quién fabrica y cuál es el origen de lo que adquiere para que su compra sea, por lo tanto, informada. Espera de sus proveedores la mejora continua de sus procesos y que repliquen en su cadena de suministro sus mismas prácticas, consciente de que cualquier ineficiencia del proveedor se refleja, en última instancia, en el servicio. Este aspecto está siendo cada vez más valorado como "calidad percibida» por los consumidores, que pueden comprobar quién es realmente el fabricante del producto consumido y establecer una relación entre el fabricante y la calidad obtenida (Calvo Porral \& Levy-Mangin, 2017). 


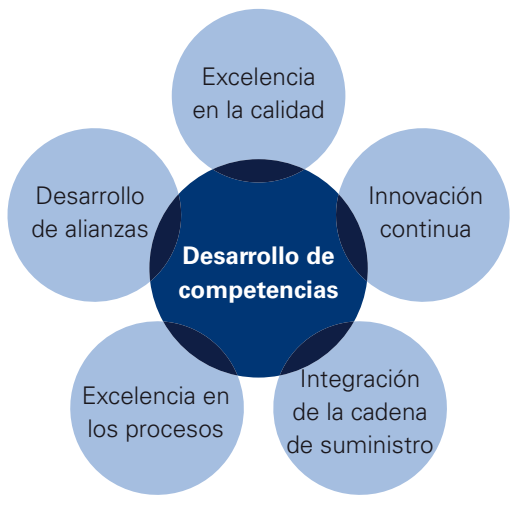

Fuente: elaboración propia.

Los interproveedores deben superar auditorías periódicas, acordadas dentro de los planes de desarrollo con Mercadona. Como parte de la política de «innovación transversal» se comprometen a realizar importantes inversiones en nuevas instalaciones y en la mejora de procesos. Así, en el año 2015, los interproveedores destinaron $€ 525$ millones a investigación y desarrollo, y alrededor del $2 \%$ de su personal a actividades de investigación, desarrollo e innovación, porcentaje superior a la media española.

Estas inversiones se deben, en gran medida, a la decisión de producir localmente aquellos productos que hasta ahora se adquirían en el extranjero. Solo cuando, por problemas de requerimientos o de capacidad de abastecimiento, no consiguen los productos en España, se recurre a importaciones (por ejemplo, solo un 3\% de las peras y un $14 \%$ de las cebollas son importadas). Citemos a título de ejemplo los $€ 70$ millones invertidos en el año 2015 por el Grupo Siro y por Queserías Entrepinares en la apertura de una factoría de barritas de cereales y de diversas líneas de producción de queso para untar, respectivamente, que han generado 170 puestos de trabajo.

Los acuerdos de colaboración se basan en seis principios, que son respetados por ambas partes: a) objetivos conjuntos y compartidos, aspecto ya mencionado como característica diferenciadora que genera valor adicional en el éxito de las alianzas; b) existencia de sinergias entre ambas compañías, buscando la "máxima eficiencia»; c) acuerdos beneficiosos para ambas partes; d) compromiso total de cumplimiento de los compromisos y plazos; e) transparencia para aumentar la productividad y eficiencia, usando sistemas de control financiero tipo open book; y f) relación estable, con acuerdos indefinidos solo cancelables por problemas relacionados con el no cumplimiento de los compromisos de calidad u objetivos mutuos. En cualquier caso, de darse una ruptura de la relación, existe un plazo de rescisión/desenganche acordado por ambas partes (Mercadona e interproveedor) que puede llegar hasta los tres años.

Como resultado de todo esto, el conjunto de los interproveedores de la compañía forma un potente clúster industrial, con más de 47000 trabajadores y 240 fábricas. Todo ese clúster ha creado una potente red de relaciones colaborativas con Mercadona que lo convierte en un potente motor para la mejora de los procesos de su cadena de suministro, habiendo desarrollado unas prácticas de mejora continua muy superiores a las que se encuentran en otras compañías de la industria. 


\subsection{Prácticas medioambientales}

Dentro del marco del concepto de sostenibilidad, tal como ha sido definido al inicio de este documento, Mercadona y su red de interproveedores trabajan desde dos ángulos: a) minimizar su impacto en términos de optimización del reciclaje de residuos; y b) otorgar especial importancia a las prácticas relacionadas con la reducción de los "desperdicios» que puedan afectar negativamente el medio ambiente y el resultado económico de la empresa. En esta área, el enfoque de la red de interproveedores de Mercadona ha sido principalmente activo, aplicando prácticas como:

- Un sistema muy avanzado de previsión de la demanda en cada tienda, que cuenta con un algoritmo que les permite gestionar el reaprovisionamiento diario a cada una de las 1600 tiendas, aproximadamente, sin que las mismas generen obsoletos significativos ni cuenten con un alto stock. La compañía registra toda la información generada por las compras de los clientes y esta información es tratada dentro de un sistema de big data para mejorar sus procesos.

- La mejora en el diseño del producto, desarrollando prácticas de «ecodiseño» tales como la reducción del espesor de los frascos de cristal, manteniendo la seguridad y funcionalidad de los mismos. Son buenos ejemplos de ello el cambio en el diseño de las botellas de aceite para poder aumentar el volumen por pallet y la utilización de envases de celulosa compactada, material que reduce el volumen de las cajas y disminuye los residuos.

- La mejora en el proceso productivo, potenciando y priorizando la inversión de sus interproveedores en procesos que consuman menos energía y generen menos desperdicios.

- La reutilización de los residuos que generan los interproveedores, dado que estos forman una red industrial que comparte información y colaboran entre ellos. Así, los residuos productivos de algunos proveedores son utilizados en los procesos productivos de otros, como en el caso del Grupo Siro, que ha creado una empresa que reutiliza los desperdicios que se generan en la fabricación de galletas y los aprovecha para la alimentación animal; o la empresa Bynsa, que reaprovecha el arroz partido que se genera en el interproveedor Arrocerías Pons para elaborar piensos para mascotas. En el caso de los proveedores de frutas, los limones que exceden el calibre o son de un tamaño excesivamente reducido $-\mathrm{y}$, por lo tanto, no pueden ser vendidos a los consumidores-, son aprovechados por los interproveedores de helados para la elaboración de sus productos.

- La gestión logística, apreciable en el uso del software Tetris, que permite mejorar la optimización de la carga de los camiones en el flujo entre los almacenes reguladores y las tiendas; en el diseño cuidadoso de los flujos de los camiones que reaprovisionan las tiendas para realizar logística inversa, en particular de pallets y embalajes; o en la gestión de la utilización de equipos especialmente diseñados para minimizar el ruido de la descarga nocturna en tiendas ubicadas en zonas donde la diurna sería lenta y complicada, como por 
ejemplo el diseñado utilizando muelles de elevación de pallets, que reduce el ruido y el tiempo de operación.

- Un programa especialmente desarrollado de gestión de los residuos de cartón, plástico y madera, los cuales son reciclados tras una clasificación previa en la tienda.

- Tras todas estas mejoras, cabe preguntarse por los resultados obtenidos. La disposición de indicadores muy detallados de su eficiencia les permite concluir, por ejemplo, que han reducido la distancia recorrida por $\mathrm{m}^{3}$ vendido un $17 \%$ entre 2009 y 2015 , mientras que la reducción en volumen transportado equivalente obtenida por el cambio en el diseño de las botellas de aceite ha sido de un $16 \%$ para el ítem.

\subsection{Compromiso con la sociedad}

El concepto de «compromiso con la sociedad» es algo que ha surgido en las últimas décadas. Miltron Friedman (1970) argumentaba que la única prioridad para la empresa era obtener beneficios para sus accionistas; sin embargo, autores como Freeman (1984) desarrollarían teorías relacionadas con la responsabilidad social corporativa (RSC), enfatizando la importancia de las relaciones con los grupos de interés que existen en los entornos donde operan las organizaciones. Para centrar el contexto en el que se desarrolla este compromiso, algunos autores (García del Junco, Palacios Florencio \& Espasandin Bustelo, 2014) consideran que la empresa no solo tiene que satisfacer a los accionistas, sino también a aquellos colectivos con los que tiene relación, que puedan ser afectados por su actividad y sin los cuales la empresa dejaría de existir: proveedores, clientes, comunidad, empleados, etcétera. Según autores como Calero (2012), hay ciertos rasgos que caracterizan estas relaciones: a) voluntariedad, b) integración en todas las áreas de la empresa, c) diálogo con los otros agentes del entorno en el que se encuentra la empresa, y d) transparencia en las acciones. En el caso de la red formada por Mercadona, el enfoque que dan a su aportación a la sociedad puede resumirse en el siguiente esquema, correspondiente a la información publicada en la Memoria de 2016 de la compañía (tabla 2).

Tabla 2. Impacto social de Mercadona

Programas de conciliación familiar para empleados

Impacto económico en la sociedad: 1,8 \% del PIB de España

$85 \%$ de las compras locales (España)

Inversión (Mercadona + interproveedores): € 1245 millones

Impuestos pagados: $€ 1468$ millones

Desarrollo de métodos de transporte menos contaminantes

Trabajo colaborativo con asociaciones de consumidores

Proyectos de mejora de los entornos vecinales

Programas de donación de alimentos

Socio activo del Pacto Mundial

Fuente: adaptado de Mercadona (2016). 
Su enfoque apunta a la potenciación del lado positivo que se crea en su entorno como consecuencia de su actividad por el impacto económico (creación de riqueza y empleo), la colaboración con organizaciones de ayuda a colectivos desfavorecidos y las actividades que tratan de paliar, aunque sea parcialmente, el impacto de la actividad económica sobre el medio ambiente (reducción de las emisiones de $\mathrm{CO}_{2}$, búsqueda de transportes más eficientes y menos contaminantes, diseño de tiendas más sostenibles energéticamente, etcétera).

\subsection{Implicación de los empleados}

Otro de los pilares de la política de Calidad Total de la empresa son los empleados. Las aproximadamente 79000 personas que trabajaban en la empresa a finales de 2016 lo hacían en un ambiente muy diferente al existente en el resto de cadenas de distribución españolas que venden gamas de productos similares.

La norma general del sector minorista en todos los países es conseguir la mayor flexibilidad posible a través de las personas que trabajan en sus establecimientos, con horarios que cambian continuamente, contratos temporales y bajos salarios. En Mercadona la situación es muy diferente ya que prácticamente todos sus empleados tienen contrato fijo, reciben un mes de formación al entrar en la empresa, se busca la conciliación familiar (por ejemplo, al no atender los domingos) y, además, sus salarios están por encima de la media de la industria. No buscan personas que hacen un trabajo rutinario en una función concreta (Harter, Schmidt \& Hayes, 2002), sino personas polivalentes que se puedan convertir en "prescriptores», de tal forma que conozcan los productos y aconsejen a los clientes cómo utilizarlos mejor. Entrenan a los empleados para conseguir que la escucha al cliente sea una de las prácticas más beneficiosas de la empresa, aplicable a la mejora de sus productos y procesos. Asimismo, fomentan la aplicación de estas prácticas en sus interproveedores de una forma activa.

A los empleados se les instruye en el modelo de CalidadTotal, usando herramientas internas elaboradas por ejecutivos de la propia compañía con el fin de que los nuevos empleados conozcan desde el primer momento los principios y valores que impregnan el conjunto de la organización, con énfasis en la eficiencia y el enfoque al cliente. Estas prácticas son muy similares a las estudiadas en las organizaciones que buscan la excelencia a partir de las prácticas de lean operations en organizaciones donde la calidad de sus procesos es el principal eje estratégico (Bowen \& Lawler, 1992). La cultura del esfuerzo y el mérito es la base de la organización, y la empresa es capaz de invertir las cuatro primeras semanas de trabajo de cada nuevo empleado en formarle para que lo haga bien, con un coste estimado de $€ 5000$ por persona (Ton \& Harrow, 2010). Asimismo, la compañía suele promover a los trabadores a puestos superiores cuando han demostrado sus capacidades y competencias para ello, siendo inusual la contratación de ejecutivos procedentes del exterior de la empresa; por el contrario, son los trabajadores que han demostrado durante su vida laboral que reúnen las condiciones requeridas para desempeñar labores de gestión ejecutiva los que alcanzan ese nivel. Todo esto podría modelizarse en unos criterios de relación con los empleados como los incluidos en el gráfico 2. 


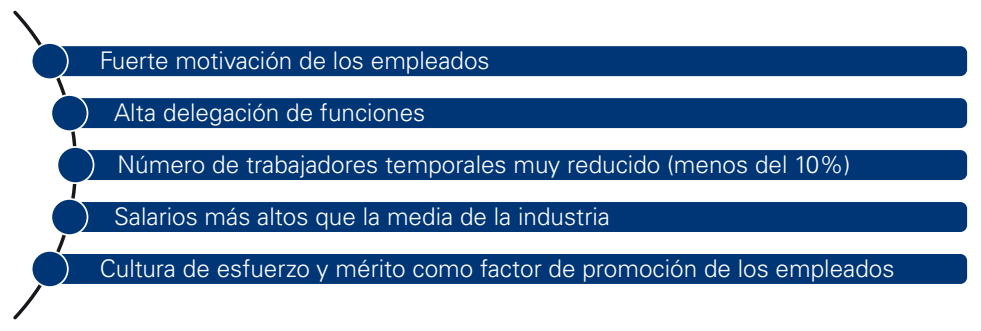

Fuente: elaboración propia.

Respecto al coste de la formación de los trabajadores, la empresa dedicó € 520 por trabajador en 2016 a la formación de los mismos (1,6 millones de horas de formación), especialmente para impulsar los nuevos modelos de venta de productos frescos y preparar a los nuevos empleados antes de que comiencen a trabajar en las tiendas.

El interés de la compañía por conocer las opiniones de los consumidores es tan alto que incluso los instructores de prescripción llegan a sentarse en los cafés cerca del establecimiento para escuchar lo que los clientes comentan sobre los productos y servicios de Mercadona (Callejo, 2012). De igual forma, la compañía lleva a cabo reuniones de prueba de productos periódicamente con decenas de miles de consumidores que año a año pasan por sus instalaciones.

En contraprestación a su involucramiento en la mejora de la compañía, los empleados conocen el salario mínimo neto que cobrarán en cada uno de sus cuatro años iniciales de trabajo en la empresa, tienen beneficios sociales superiores a los establecidos en las normas laborales —como el derecho a un mes adicional de lactancia para las madres - y se ven beneficiados por la promoción de la conciliación a través de la creación de centros educativos infantiles gratuitos en algunos bloques logísticos. La compañía también lleva a cabo prácticas que se reflejan en los estudios realizados sobre la motivación en las empresas, obteniendo uno de los primeros lugares a nivel nacional como empresa recomendada para trabajar, según los análisis realizados por Adecco (2015).

\section{Discusión y conclusiones}

Al inicio de este trabajo se propuso como objetivo dar un paso adicional hacia el estudio del modelo de sostenibilidad de la cadena de suministro de Mercadona, el minorista líder en el mercado español, considerándose que la sostenibilidad desarrollada en su cadena de suministro podría explicarse por la construcción de un "conjunto armónico» de factores que distinguen a su cadena de suministro de otras, a saber:

- $\quad$ Factores tradicionales que pueden ser la base para la formación de alianzas. También se producen en otras cadenas de suministro.

- Factores adicionales que fortalecen sus relaciones de colaboración con las empresas que los poseen (objetivos a largo plazo y objetivos compartidos).

- Utilización de prácticas de lean operations que apoyan la sostenibilidad de las cadenas de suministro en Mercadona y en su red de interproveedores. 
- Reproducción del modelo desarrollado por Mercadona en las prácticas internas de sus principales interproveedores. Con efectos multiplicadores, esta práctica retroalimenta la cadena y hace que exista un «proceso de mejora continua».

- Los factores «a» y «b» ya han sido estudiados por el autor de este estudio en los trabajos mencionados en la introducción, siendo el objetivo del presente artículo profundizar en la sostenibilidad de la cadena de suministro de Mercadona a partir del desarrollo de los factores "c» $y$ «d».

La cadena de suministro analizada liga la eficiencia a la reducción de los desperdicios de una forma muy similar a la establecida por los estudios desarrollados por los precursores de los principios del sistema de producción de Toyota (Ohno, 1988) y de mejora continua (Imai, 1986), circunstancia muy novedosa en el sector en el que desarrolla sus actividades Mercadona, donde las empresas tradicionalmente siguen la práctica de trasladar estos desperdicios (en forma de stock, obsoletos y otras ineficiencias) aguas arriba; es decir, hacía sus proveedores, esperando que las ineficiencias y sus costes relacionados sean absorbidas por ellos. El enfoque de Mercadona resulta ser muy diferente, ya que dicha política cortoplacista no mejora de ninguna manera la eficiencia presente y futura de los proveedores ni, por lo tanto, de la cadena de suministro, sino que los debilita y condiciona para mejorar su eficiencia a largo plazo, pues tienen que absorber sus propias ineficiencias, además de aquellas que generan sus clientes. Al buscar la reducción de defectos/ desperdicios, están introduciendo en toda su cadena de suministro un factor de eficiencia que tiene un claro reflejo en dos ángulos de la sostenibilidad: el ángulo económico y el ángulo medioambiental.

De acuerdo al análisis del modelo operativo de la empresa, este gira en torno al cliente y centra todos los esfuerzos en que este sienta que va a conseguir el mejor precio del mercado, así como precios estables, un nivel de calidad alto y una gama razonablemente amplia de productos. Todo el modelo se soporta en principios conceptuales desarrollados en torno a un "conjunto armónico de buenas prácticas», como:

- La gestión excelente de las operaciones, que habitualmente se han incluido bajo la denominación de lean operations practices, hasta ahora ampliamente aplicadas en empresas de sectores industriales, pero en mucho menor medida en empresas de servicios. Resulta interesante comprobar que estas prácticas pueden ser empleadas con notable éxito en empresas de este sector, como lo demuestra su aplicación en la empresa estudiada. Quizá un factor clave en el éxito, que se infiere a partir de las entrevistas con los ejecutivos, sea la absoluta involucración de la más alta dirección de las empresas que forman parte de la cadena de suministro en el detalle de cómo se ejecutan las operaciones y en el servicio al cliente. Esta podría ser una de las razones que explican la robustez y consistencia de la política de "cero defectos".

- La importancia destinada a la reducción y mitigación del impacto ambiental que se provoca a consecuencia del negocio, tanto por el compromiso que 
implica con la sociedad y el futuro como por la repercusión que tiene en la eficiencia de las operaciones de la propia organización.

- El desarrollo de alianzas como una forma de utilizar todas las capacidades y competencias de los miembros de la cadena de suministro para conseguir hacer más, incluso con menos recursos. El modelo está fuertemente soportado por el grupo de 126 interproveedores, que aportan su innovación y capacidad industrial. Esta circunstancia se ve reforzada por la extensión de los acuerdos de colaboración en la cadena de suministro a los productores de materias primas (cereales, carne, etcétera) con la pretensión de alcanzar una "cadena sostenible integral» $y$, de esa forma, garantizar la seguridad alimentaria, la estabilidad de los precios y el mantenimiento de un flujo estable de productos (hacía el consumidor) y rentas (hacía todos los miembros de la cadena de suministro).

- Todo lo anterior se ve apoyado por una gestión de personas muy diferente a la clásica en el sector minorista, donde no se busca "optimizar el uso» de las personas como «recursos» con métodos basados en una flexibilidad extrema, sino mantener un equilibrio entre la flexibilidad, la polivalencia y la conciliación personal, además de usar la motivación del personal como un factor para la satisfacción del cliente y la mejora continua.

- A partir de estos elementos es posible inferir el modelo estratégico de la cadena de suministro (gráfico 3) que está permitiendo a Mercadona ser el líder de la distribución minorista en España, con una participación del 24\% del mercado español, muy superior a sus principales competidores (Carrefour, Día, El Corte Inglés, etcétera). Sin duda, el modelo es sólido y entre las más importantes razones de esta solidez podemos mencionar el hecho de que sus objetivos y estrategia se mantienen en el tiempo, que hay un equipo directivo liderado por una propiedad familiar estable y que, al no cotizar en bolsa, la compañía puede permitirse adoptar ciertas prácticas buscando resultados a largo plazo, aunque a corto plazo no consigan obtener beneficios directos de las mismas.

Gráfico 3. Modelo estratégico en la cadena de suministro de Mercadona

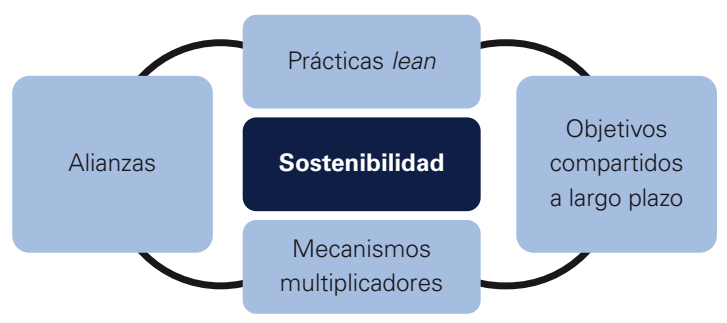

Fuente: elaboración propia.

La de Mercadona es una cadena de suministro gestionada por ejecutivos que no buscan resultados a corto plazo y han entendido desde el principio que su estrategia está soportada por su red de proveedores clave (interproveedores), los cuales han ligado 
en gran medida su futuro al de sus clientes. Aquí está otro de los grandes elementos de cohesión del modelo, ya que el negocio y su futuro está construido sobre relaciones de colaboración a largo plazo («de por vida») tipo alianzas que, a su vez, contienen elementos de compromiso a largo plazo y una visión compartida que las vuelven más duraderas y sólidas que las relaciones establecidas por otras empresas. Todo esto tiene mucho que ver con una visión estratégica a largo plazo que no es frecuente encontrar en la práctica empresarial, y constituye un marco real donde se reflejan gran parte de los modelos conceptuales teóricos desarrollados para tratar de modelizar la colaboración en la cadena de suministro. Además, el conjunto de estos interproveedores forma una red industrial muy potente y única en la industria alimentaria europea, siendo su objetivo primario conseguir una cadena de suministro cada vez más eficiente y enfocada en el cliente final, cuyos requerimientos están siempre basados en la mejora continua (eliminación permanente del desperdicio) de la totalidad de la cadena. En simultáneo, cada uno de los interproveedores replica las prácticas de la empresa focal (Mercadona), originándose de esta forma un efecto multiplicador de mejora en el conjunto de la cadena de suministro.

A partir del análisis realizado, se puede inferir que el «modelo estratégico» de esta cadena de suministro (figura 3) cumple con los ángulos de la sostenibilidad integral (económica, social y medioambiental) en el sentido establecido por el triple bottom line de Elkington. En ese sentido, consigue cubrir de forma amplia, desde la cadena de suministro sostenible, los requerimientos establecidos en la literatura para que esta sea considerada como tal, al nivel de otras compañías internacionales que durante años han buscado $-\mathrm{y}$ conseguido- ser reconocidas como empresas con cadenas de suministro sostenibles.

Podría sugerirse que las prácticas descritas constituyen un modelo de buenas prácticas aplicable a otras empresas del mismo sector o de diferentes sectores empresariales. Sin embargo, las compañías que consideren su aplicación como modelo integral deberán estudiar cuidadosamente su encaje, considerando sus características específicas y circunstancias, ya que el modelo estratégico de esta cadena de suministro incluye tanto prácticas de probable aplicación en otras cadenas de suministro (como las relativas a la eliminación sistemática de defectos) como otras que dan consistencia al modelo y que son difícilmente exportables a organizaciones distintas a las que las han desarrollado (como el modelo de relación colaborativa con los interproveedores, la búsqueda de resultados a largo plazo o la generación de mecanismos de replicación del modelo de la empresa focal en la totalidad de la cadena de suministro). 


\section{bibliografía}

Adecco

2015

III Encuesta sobre mejores empresas y

jefes más felices para trabajar. Disponible

en: <http://www.adecco.es/_data/

NotasPrensa/pdf/652.pdf > .

\section{Bowen, D. E. \& E. E. Lawler}

1992 Total quality-oriented human resources management. Organizational Dynamics, 20(4), 29-41. Recuperado de: <https://doi. org/10.1016/0090-2616(92)90073-V> .

\section{Brundtland Commision}

World Commission on Environment and Development. Environmental Policy and Law. Nueva York: United Nations.

\section{Callejo, M. B.}

Using Total Quality Management Model to Face the Economic Crisis: The Case of Mercadona (pp. 36-58). INTECH Open Access Publisher.

\section{Calvo Porral, C. \& \\ J. P. Levy-Mangin}

2017

Specialty food retailing: Examining the role of products' perceived quality. British Food Journal, 119(7), 35-49. Recuperado de: <https://doi.org/10.1108/BFJ-11-2016$\underline{0567>}$.

\section{Carter, C. R. \& D. S. Rogers}

A framework of sustainable supply chain management: Moving toward new theory. International Journal of Physical Distribution \& Logistics Management, 38(5), 360387. Recuperado de: <https://doi. org/10.1108/09600030810882816> .

\section{Cooper, M. C.; K. M. Ellram;}

J.T. Gardner \& A. M. Hanks

Meshing multiple alliances. Journal of Business Logistics, 18(1), 67-82.

\section{Coyle, J. J.; C. J. Langley;}

\section{R. A. Novack \& B. Gibson}

2016 Supply Chain Management: A Logistics Perspective. Londres: Nelson Education.

\section{Chopra, S. \& P. Meindl}

2007 Supply chain management. Strategy, planning \& operation. En C. Boersch y R. Elschen (eds.), Das Summa Summarum des Management (pp. 265-275).

Gabler. Recuperado de: <https://doi. org/10.1007/978-3-8349-9320-5_22>.

\section{Christopher, $\mathbf{M}$.}

Logistics \& Supply Chain Management. Londres: Pearson.

\section{De la Calle, Vicente A.;}

\section{A. Barinaga \& J. C. Gietz}

La colaboración como estrategia en la cadena de suministro: una visión metodológica. Dyna Management, 4, 23-37. Recuperado de: <https://doi. org/10.6036/mn7809>

Dubey, R.; A. Gunasekaran, T. Papadopoulos, S. J. Childe,

\section{K.T. Shibin \& S. F. Wamba}

Sustainable supply chain management:

Framework and further research

directions. Journal of Cleaner

Production, 142, 1119-1130. Recuperado de: $<$ https://doi.org/10.1016/i.

jclepro.2016.03.117>. 


\section{Durham, Y.}

2000

An experimental examination of

double marginalization and vertical

relationships. Journal of Economic

Behavior \& Organization, 42(2), 207-229.

Recuperado de: <https://doi.org/10.1016/

$\underline{\text { S0167-2681(00)00086-X }>\text {. }}$

\section{Eisenhardt, K. M.}

1989

Building theories from case study research.

Academy of Management Review, 14(4),

532-50. Recuperado de: <https://doi.

org/10.5465/amr.1989.4308385>.

\section{Ehrenfeld, J.}

Towards the sustainable corporation: Win-

win-win business strategies for sustainable

development. California Management

Review, 36(2), 90-100. Recuperado de:

$<\underline{\text { https://doi.org/10.2307/41165746> }}$.

\section{Ehrenfeld, J.}

2004

Industrial ecology: A new field or only a

metaphor? Journal of Cleaner Production,

12(8), 825-31. Recuperado de: <https://doi.

org/10.1016/i.jclepro.2004.02.003>

\section{Elkington, J.}

Partnerships from cannibals with forks: The triple bottom line of $21 \mathrm{st}$ \century business.

Environmental Quality Management,

1(1), 37-51. Recuperado de: <https://doi.

org/10.1002/tgem.3310080106>.

\section{Henriques, A. \& J. Richardson}

Enter the triple bottom line. En A.

Henriques y J. Richardson (eds.), The Triple

Bottom Line: Does It All Add Up? Londres:

Earthscan.

\section{Forrester, J. W.}

Industrial dynamics: A major breakthrough

for decision makers. Harvard Business

Review, 36(4), 37-66.
Freeman, R. E.

1984

Strategic Management: A Stakeholder

Approach. Boston: Printman Press.

\section{Friedman, $\mathbf{M}$.}

The Counter-Revolution in Monetary

Theory: First Wincott Memorial Lecture, Delivered at the Senate House, University of London, 16 September, 1970 (XXXIII). Institute of Economic Affairs.

\section{Gaudin, G.}

2016

Pass-through, vertical contracts, and bargains. Economics Letters, 139, 1-4. Recuperado de: <https://doi.org/10.1016/i. econlet.2015.11.043>.

\section{García del Junco, J.;}

\section{B. Palacios Florencio \&}

\section{F. Espasandin Bustelo}

2014

Manual práctico de responsabilidad social corporativa. Gestión, diagnóstico, e impacto de la empresa en la empresa. Madrid: Pirámide.

\section{Govindan, K.; S. G. Azevedo,}

\section{H. Carvalho \& V. Cruz-Machado}

2014 Impact of supply chain management practices on Sustainability. Journal of Cleaner Production, (85), 212-225. Recuperado de: <https://doi.org/10.1016/j. jclepro.2014.05.068>.

\section{Gummesson, E.}

2000 Qualitative Methods in Management Research. Londres: Sage. 


\section{bibliografía}

Harter, J. K.; F. L. Schmidt \&

\section{T. L. Hayes}

2002

Business-unit-level relationship between

employee satisfaction, employee

engagement, and business outcomes:

A meta-analysis. Journal of Applied

Psychology, 87(2), 268-285. Recuperado

de: <https://doi.org/10.1037/0021-

$\underline{9010.87 .2 .268>}$

Helleno, A. L.; A. J. I. de Moraes

\section{\& A.T. Simon}

2017

Integrating Sustainability indicators

and Lean Manufacturing to assess

manufacturing processes: Application

case studies in Brazilian industry. Journal

of Cleaner Production, (153), 405-416.

Recuperado de: <https://doi.org/10.1016/i. jclepro.2016.12.072>.

\section{Hong, G. H. \& N. Li}

Market structure and cost pass-through

in retail. Review of Economics and

Statistics, 99(1), 151-166. Recuperado de:

<https://doi.org/10.1162/REST_a_00560>

\section{Imai, $\mathbf{M}$.}

Kaizen. Nueva York; Londres: Random

House Business Division.

\section{Ketokivi, M. \& T. Choi}

Renaissance of case research as a scientific method. Journal of Operations Management, 32(5), 232-240

Recuperado de: <https://doi.org/10.1016/j. jom.2014.03.004>.

\section{Kolk, A.}

governance: Exploring multinationals'

reporting practices. Business Strategy and the Environment, 17(1), 1-5. Recuperado

de: <https://doi.org/10.1002/bse.511>

\section{La Londe, B. J. \& J. M. Masters}

1994 Emerging logistics strategies: Blueprints

for the next century. International

Journal of Physical Distribution \&

Logistics Management, 24(7), 35-

47. Recuperado de: <https://doi.

org/10.1108/09600039410070975>.

Lambert, D. M. \& M. C. Cooper

2000

Issues in supply chain

management. Industrial Marketing

Management, 29(1), 65-83. Recuperado

de: <https://doi.org/10.1016/S0019-

8501(99)00113-3>

\section{Mangan, J. \& C. Lalwani}

2016 Global Logistics and Supply Chain Management. Londres: John Wiley \& Sons.

\section{Mercadona}

2016 Memoria anual. Recuperado de: <https:// www.mercadona.es/document/es/

memoria-anual-2016.pdf>.

Mentzer, J. T.; W. DeWitt, J. S. Keebler, S. Min, N. W. Nix, C. D. Smith \& Z. G. Zacharia

Defining supply chain

management. Journal of Business

logistics, 22(2), 1-25. Recuperado de:

$<$ https://doi.org/10.1002/j.2158-1592.2001.

tb00001.x>

\section{Miguel B. C. \& G. B. Santiago}

2010

Application of the total quality

management approach in a Spanish

retailer: The case of Mercadona. Total 


\section{bibliografía}

Quality Management, 21(12), 1365-1381.

Recuperado de: <https://doi.org/10.1080/14 783363.2010.530782>

\section{Morcillo, J.; A. Díaz \& L. Solís}

2015

Improving alliances: Insights from the practices of successful companies. The IUP Journal of Supply Chain Management, 12(3), 50-61.

\section{Morcillo, J. \& A. Duran}

2016

Explaining alliance success factors in Spanish food and beverage supply chain: Case analysis. En Engineering Systems and Networks (pp. 167-174). Cham, Baviera: Springer. Recuperado de: <https:// doi.org/10.1007/978-3-319-45748-2 18>.

\section{Morcillo, J. \& A. Duran}

Supply chain sustainability in Spanish major retailer through strategic alliances and lean practices. En Closing the Gap between Practice and Research in Industrial Engineering (pp. 11-18). Cham, Baviera: Springer.

\section{Ohno, T.}

1998 Toyota Production System: Beyond LargeScale Production. Florida: CRC Press.

\section{Rother, M.}

Toyota Kata: el método que ayudó a miles de empresas a optimizar la gestión de sus negocios. Barcelona: Profit Editorial.

\section{Shah, R. \& P.T. Ward}

Lean manufacturing: Context, practice bundles, and performance. Journal of Operations Management, 21(2), 129-149. Recuperado de: <https://doi.org/10.1016/ $\underline{\text { S0272-6963(02)00108-0>. }}$.

\section{Sheffi, Y.}

2005

The Resilient Enterprise: Overcoming Vulnerability for Competitive Advantage. Cambridge, Massachusetts: MIT Press.

\section{Sindi, S. \& M. Roe}

2017

Strategic Supply Chain Management: The Development of a Diagnostic Model. Cham, Baviera: Springer.

\section{Tan, B. I.; K. L. Wong \& C. K. Choong}

2015

Can TQM improve the Sustainability of family owned business? International Journal of Innovation and Learning, 17(2), 174-186. Recuperado de: <https://doi. org/10.1016/S0272-6963(02)00108-0>

\section{Wee, H. M. \& S. Wu}

2009

Lean supply chain and its effect on product cost and quality: A case study on Ford Motor Company. Supply Chain Management: An International Journal, 14(5), 335-41. Recuperado de: <https://doi. org/10.1108/13598540910980242>.

Womack, J. P. \& D. T. Jones

Lean Thinking: Banish Waste and Create Wealth in Your Corporation. Nueva York: Simon and Schuster.

\section{Womack J.P.; D. T. Jones \& D. Roos}

The Machine that Changed the World. Nueva York: Simon and Schuster.

\section{Ton, Z. \& S. Harrow}

Yin, R. K. Methods. Londres: Sage. 